\title{
INTOXICAÇÕES EXÓGENAS AGUDAS REGISTRADAS EM CENTRO DE ASSISTÊNCIA TOXICOLÓGICA
}

\section{Marina Moura Toscano}

Discente de Medicina na Universidade Federal da Paraíba (UFPB), Brasil.

E-mail:marinamtoscano3@hotmail.com

Jersica Tamara Amorim Landim

Graduada em Medicina na Universidade Federal da Paraíba (UFPB), Brasil.

\section{Artur Bastos Rocha}

Graduado em Medicina na Universidade Federal da Paraíba (UFPB), Brasil.

\section{Rilva Lopes de Sousa-Muñoz}

Doutora pela Universidade Federal da Paraíba (UFPB), Docente associada do Departamento de Medicina Interna da Universidade Federal da Paraíba (UFPB), Brasil.
RESUMO: O objetivo deste trabalho foi descrever o perfil clínico e sociodemográfico dos casos de intoxicação aguda registrados pelo Centro de Assistência Toxicológica da Paraíba (Ceatox-PB) em 2012. O estudo foi transversal e retrospectivo, pautado na análise documental de dados secundários obtidos no Ceatox (PB). No ano de 2012, foram atendidos 208 casos de intoxicações exógenas no Ceatox-PB. A idade dos pacientes variou de 1 a 77 anos $(17,3 \pm 18)$, com faixa etária mais prevalente inferior a 18 anos $(58,2 \%)$, e $52,8 \%$ do sexo feminino. $O$ principal tipo de intoxicação foi acidente individual (47,6\%), e no grupo entre 20-40 anos os casos relacionaram-se a tentativas de suicídio e acidentes individuais. As tentativas de suicídio foram mais frequentes no sexo feminino $(60,8 \%)$. Os casos ocorreram no ambiente domiciliar (80\%) e as causas mais frequentes foram medicamentos $(24,6 \%)$ e via de intoxicação oral $(83,2 \%)$. O padrão epidemiológico das intoxicações exógenas notificadas pelo Ceatox-PB, em 2012, foi similar ao relatado em outros estudos, em que jovens e mulheres foram os grupos de maior risco e medicamentos ingeridos de forma acidental, os principais causadores.

PALAVRAS-CHAVE: Centros de Controle de Intoxicações; Envenenamento; Estudos Epidemiológicos.

\section{ACUTE EXOGENOUS INTOXICATIONS REPORTED IN A TOXICOLOGICAL CARE CENTER}

\begin{abstract}
Clinical and socio-demographic profile of acute intoxication cases reported at the Toxicological Care Center of Paraíba (Ceatox-PB), Brazil, in 2012, is described by a transversal and retrospective study based on the documental analysis of secondary data retrieved from Ceatox-PB. In 2012, 208 cases of exogenous intoxications were treated at Ceatox-PB. Patients ranged between 1 and 77 years $(17,3 \pm 18)$, with prevalent age bracket lower than 18 years $(58,2 \%) ; 52,8 \%$ were female. Main intoxication was accidental (47,6\%), and in groups aged 20 - 40 years related to suicide attempts and individual accidents. Attempts at suicide were more frequent in females $(60,8 \%)$. Cases occurred at home $(80 \%)$ and medicines taken orally $(83,2 \%)$ were the most frequent cause $(24,6 \%)$. Epidemiological pattern of exogenous intoxications at Ceatox-PB in 2012 was similar to that in other studies where young people and females constituted the highest risk group, with main agent as accidentally-taken medicines.
\end{abstract}

KEY WORDS: Poison Control Centers; Poisoning; Epidemiologic studies. 


\section{INTRODUÇão}

Intoxicação exógena é a manifestação, através de sinais e sintomas, dos efeitos nocivos produzidos em um organismo vivo como resultado da sua interação com alguma substância química, que pode ser encontrada no ambiente (água, alimentos, plantas, animais peçonhentos) ou isoladas (pesticidas, medicamentos, produtos de uso industrial, produtos de uso domiciliar) (JESUS et al., 2012).

Os casos de intoxicação exógena desempenham um papel importante no contexto dos acidentes em geral, por sua alta frequência, custos de tratamento, possibilidade de sequelas irreversíveis e sofrimento que causam às vítimas e suas famílias (MARTINS et al., 2006). Dentro desta problemática, que representa um grave problema de Saúde Pública, verificam-se diferenças acentuadas nos padrões de intoxicações entre os centros de informação toxicológica de vários países e, nestes, há diferenças regionais (AL-BARRAQ; FARAHAT, 2011; KUMAR et al., 2010).

Nos países desenvolvidos, a incidência anual de intoxicação humana, tanto não-intencional quanto deliberada, varia entre 0,2 e 0,9 por 1.000 pessoas, taxa que continua aumentando progressivamente. Atualmente um em cada 100 admissões hospitalares deve-se a intoxicações e oito em cada 100 autópsias realizadas no mundo são por morte tóxica (JIMENÉZ; KUHN, 2009).

Nos centros de assistência toxicológica, ocorre atendimento diário a dezenas de pacientes afetados, assim como o treinamento de estudantes da área de saúde, sistematização de condutas, referenciação e contrarreferenciação, além de pesquisas e divulgação de experiências clínicas. No estado da Paraíba, o Centro de Informação Toxicológica (Ceatox) é um serviço do Centro de Ciência da Saúde (CCS), ligado ao Departamento de Ciências Farmacêuticas, existente desde 1986, e atua no processo de qualificação do aprendizado dos alunos de graduação em áreas referentes à Toxicologia, temática geralmente excluída dos currículos médicos no Brasil (SILVA et al., 2015). Durante seus 20 anos de operação, o Ceatox-Paraíba tem sido uma referência na gestão do paciente com intoxicação.

O objetivo deste estudo foi descrever o perfil clínico e sociodemográfico dos casos de intoxicações agudas registradas pelo Centro de Assistência Toxicológica da Paraíba (Ceatox-PB), na cidade de João Pessoa, Paraíba, no ano de 2012.

\section{METODOLOGIA}

Trata-se de um estudo transversal, retrospectivo e de abordagem quantitativa, pautado na análise documental de dados secundários obtidos através do preenchimento das Fichas de Notificação e Atendimento ao Centro de Assistência Toxicológica da Paraíba (Ceatox-PB), João Pessoa, Paraíba, Brasil. Estas fichas são formulários padronizados de notificação do Sistema Nacional de Informações Tóxico-Farmacológicas do Ministério da Saúde.

O Ceatox-PB é um órgão de assessoria para informação de procedimentos em urgências toxicológicas aos profissionais de saúde, e ao qual são requisitadas condutas diagnósticas e terapêuticas para casos de intoxicação na cidade de João Pessoa, região metropolitana e cidades vizinhas. O Estado da Paraíba possui uma área de $53.439 \mathrm{~km}^{2}$ com 223 municípios, situado na região Nordeste do Brasil com população estimada até o ano de 2010 em 3.766.528 habitantes (SARAIVA et al., 2012).

A amostra foi recrutada retrospectivamente pelas fichas de notificação preenchidas no período de 01 de janeiro a 31 de dezembro de 2012, e que possuíam registro de intoxicações exógenas agudas, ou seja, que ocorreu ou se desenvolveu após uma única exposição (ou múltiplas nas últimas 24 horas) por um agente químico. Foram excluídas as fichas de notificação contendo casos de acidentes por animais peçonhentos e aquelas com informações incompletas.

As informações foram coletadas dos registros do banco de dados do Ceatox-PB, sendo incluídas todas as fichas de ocorrência toxicológica de pessoas de todas as idades, independente do sexo, e intoxicação pelos diversos agentes. Para investigação dos casos, foram preenchidos em uma planilha pré-codificada, os dados das fichas de ocorrência toxicológica resultante de notificação espontânea dos profissionais de saúde.

Para todas as fontes de dados, as variáveis foram classificadas em dois grandes grupos: as relacionadas 
ao indivíduo (sexo, faixa etária); e as relacionadas à intoxicação (circunstância, tipo de exposição, classe do agente toxicológico, local de exposição, causa, apresentação clínica, desfecho). O local da ocorrência foi categorizado em domicílio e ambiente externo (via pública, ambiente de trabalho e escola. A variável faixa etária foi categorizada nos seguintes intervalos: até 1 ano; 1-5 anos; 5,1-10 anos; 10,1-20 anos; 20,1-30 anos; 30,140 anos; 40,1-50 anos; 50,1-60 anos; 60,1-70 anos; 70,180 anos; acima de 80 anos.

Para análise estatística, foi utilizado o pacote estatístico SPSS (Statistical Package for the Social Sciences), versão 20.0. Os dados obtidos referentes às variáveis sociodemográficas e as variáveis nominais foram descritos através de frequências absolutas e relativas.

Estapesquisafoiaprovada peloComitêde Éticaem Pesquisa do HULW/UFPB (CAAE: 57681916.8.0000.5183; parecer 1.662.273). Por tratar-se de estudo com utilização de dados secundários, a pesquisadora responsável assinou o "Termo de Compromisso para Utilização de Dados de Fichas de Notificação do Ceatox-PB", assegurando o anonimato das informações.

\section{RESULTADO}

No ano de 2012, foram atendidos 208 casos de intoxicações exógenas no Ceatox-PB, após a exclusão de 14 fichas por informação incompleta. A idade dos pacientes incluídos variou de 1 a 77 anos (17,3 \pm 18), $52,8 \%$ do sexo feminino. A faixa etária mais prevalente foi a de indivíduos com idade inferior a 18 anos (58,2\%), seguida pela de 18 a 39 anos (Figura 1).

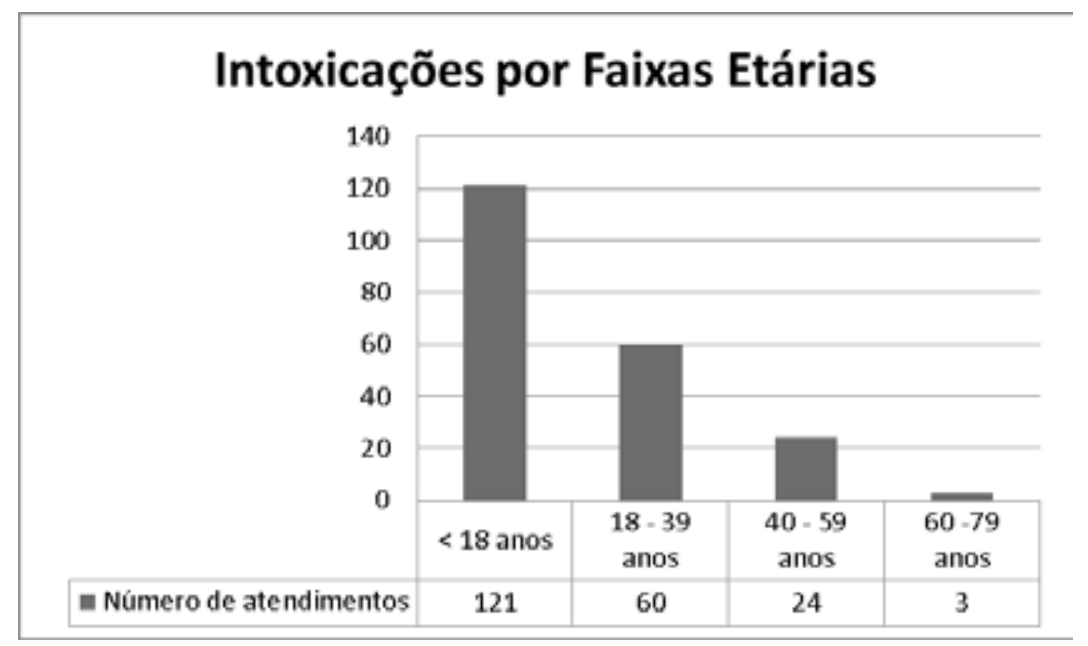

Figura 1. Intoxicações exógenas registradas pelo Ceatox-PB no município do João Pessoa durante o ano de 2012

Observou-se que a principal circunstância responsável por esses agravos foi do tipo acidente, não deliberado, que representou $71,1 \%$ dos casos (Tabela 1 ). 
Tabela 1. Circunstâncias relacionadas às intoxicações exógenas registradas pelo Ceatox-PB no município do João Pessoa durante o ano de $2012(\mathrm{n}=208)$

\begin{tabular}{lcc}
\hline \multirow{2}{*}{ Circunstâncias } & \multicolumn{2}{c}{ Frequências } \\
\cline { 2 - 3 } & $f$ & $\%$ \\
\hline Acidente individual & 137 & 65,9 \\
Tentativa de suicídio & 51 & 24,5 \\
Acidente ambiental & 4 & 1,9 \\
Acidente ocupacional & 4 & 1,9 \\
Acidente coletivo & 3 & 1,4 \\
Uso terapêutico & 3 & 1,4 \\
Abuso & 2 & 1,0 \\
Outros & 4 & 2,0 \\
\hline
\end{tabular}

Fonte: Dados da pesquisa

Na faixa etária inferior a 18 anos, o principal tipo de intoxicação foi o acidente individual, observado em $47,6 \%$ dos casos registrados, enquanto no grupo de adultos jovens entre 20-40 anos, os casos de intoxicação foram relacionados a tentativas de suicídio (12\%), seguidas por acidentes individuais (11,5\%). As tentativas de suicídio foram mais frequentes no sexo feminino (60,8\% destes casos).
O local onde ocorreu a intoxicação foi o ambiente domiciliar em $80 \%$ dos casos, seguido pelo ambiente de trabalho $(8,3 \%)$ e ambientes externos, escolas/creches e serviços de saúde $(5,8 \%)$.

As causas mais frequentes foram intoxicações por medicamentos (24,6\%) (Tabela 2).

Tabela 2. Classes de agente toxicológico relacionadas às intoxicações exógenas registradas pelo Ceatox (PB) no município do João Pessoa durante o ano de $2012(\mathrm{n}=208)$

\begin{tabular}{lcc}
\hline \multirow{2}{*}{ Classes dos agentes toxicológicos } & & Frequências \\
\cline { 2 - 3 } & $f$ & $\%$ \\
\hline Medicamentos & 51 & 24,6 \\
Agrotóxicos & 35 & 16,9 \\
Produtos químicos industriais & 34 & 16,3 \\
Produtos domissanitários & 31 & 14,9 \\
Raticidas & 26 & 12,5 \\
Plantas tóxicas & 14 & 6,8 \\
Drogas de abuso & 5 & 2,4 \\
Cosméticos & 4 & 1,9 \\
Metais & 4 & 1,9 \\
Outros & 4 & 1,8 \\
\hline
\end{tabular}

Fonte: Dados da pesquisa 
$\mathrm{Na}$ maioria dos casos, a via de intoxicação foi a oral $(83,2 \%)$, seguida pela via respiratória $(7,7 \%)$, cutânea (4,4\%), ocular (1\%), enquanto 3,7\% não tinham especificação da via nas fichas.

Verificou-se que 59,4\% dos pacientes estavam assintomáticos. A apresentação clínica registrada em 40,6\% incluiu náuseas e vômitos (42\%), sintomas inespecíficos (23\%), dor abdominal (15\%), cefaleia $(9,4 \%)$, irritação de mucosas (5\%), edema (4\%) e sintomas inespecíficos (23\%).

O desfecho clínico não foi registrado em 40,8\% dos casos, enquanto $33,7 \%$ tiveram cura não confirmada, $22,6 \%$ receberam alta e apenas $2,9 \%$ tiveram evolução para óbito.

Não foi possível avaliar as variáveis escolaridade, ocupação e relação da intoxicação com o trabalho, pois estas informações não são consolidadas no banco de dados do Ceatox-PB.

\section{DISCUSSÃO}

A maioria dos casos de intoxicação notificados ao Ceatox-PB em 2012 foi relacionada a medicamentos consumidos de forma acidental, mais frequentemente por mulheres e menores de 18 anos. A prevalência do sexo feminino apresenta concordância com outros dados brasileiros (BARRETO et al., 2015; BERTASSO-BORGES et al., 2010; MORAIS et al., 2008) e estrangeiros (ALBARRAQ; FARAHAT, 2011; KUMAR et al., 2010), porém há também estudo com relato de predominância masculina (ANTHONY, 2012). Refere-se, porém, no Brasil, prevalência de 32,6\% de intoxicações em indivíduos abaixo de 20 anos, que foi inferior à frequência encontrada no nosso estudo.

Os medicamentos ocupam o primeiro lugar entre todos os tipos de agentes tóxicos registrados pelos centros toxicológicos do Estado de São Paulo, Brasil, onde estes eventos são registrados por telefone a partir de hospitais em $78,5 \%$ dos casos, originados de exposições agudas, por via oral $(90,2 \%)$, ocorridas em residência (85,7\%), com predomínio do sexo feminino (59\%) e maior concentração na primeira década de vida $(49,4 \%)$, sobretudo nos dois e três anos de idade (GANDOLFI,
ANDRADE, 2006), de forma similar ao que foi observado no presente estudo.

A alta prevalência de intoxicações no ambiente domiciliar também é condizente com levantamento feito por Sousa Filho et al. (2009), na cidade de Sobral, Ceará, Brasil, onde 83,3\% dos casos ocorreram nos domicílios. Tendo em vista a faixa etária mais prevalente inferior a 18 anos, os acidentes no ambiente doméstico tendem a ser mais comuns. Além disso, as tentativas de suicídio, a segunda principal causa, também tendem a ocorrer em ambiente intradomiciliar. A maior prevalência do sexo feminino nas tentativas de suicídio também está em conformidade com estudos anteriores (BAHLS; BOTEGA, 2007; VIANA et al., 2008).

A principal causa de intoxicação exógena de nossa amostra também corrobora estudo feito nos anos de 2007 e 2008, em que 34,2\% dos casos foram classificados como acidentais. Também em concordância com o presente estudo, pesquisa realizada para caracterizar as intoxicações humanas notificadas ao Ceatox de Marília SP, em 2004, e que incluiu retrospectivamente 872 fichas de notificação, verificou-se que a maior percentagem de intoxicação ocorreu nas faixas etárias de 0-9 e de 20-29 anos, sendo que a faixa de 1-4 anos representou $20 \%$ de todas as intoxicações, com uma prevalência elevada das circunstâncias acidental e tentativa de suicídio, e as categorias de agentes tóxicos mais frequente, medicamentos, agrotóxicos e domissanitários (MONTEIRO; CARVALHO JUNIOR, 2007), mostrando, portanto, perfil semelhante ao encontrado no nosso estudo. A via oral foi a via de exposição mais prevalente para as intoxicações. Resultado semelhante foi observado no período de 2000 a 2004 no Estado de Minas Gerais, com porcentagem de $63 \%$ das 86 intoxicações analisadas (MOREIRA et al., 2010).

Os tipos de intoxicação no nosso estudo apoiam a crescente potencialidade para tentativas de suicídio, em que os medicamentos foram as causas mais comuns, seguidos por produtos químicos domésticos (MOREIRA et al., 2010; MONTEIRO; CARVALHO JUNIOR, 2007). A maior prevalência da intoxicação medicamentosa entre os casos de intoxicação analisados está em concordância com a literatura, em que os fármacos assumiram o papel de principal causa de intoxicações gerais (MENDONÇA; 
MARINHO, 2005). Os resultados do presente estudo corroboram pesquisa anterior, em que entre as causas de envenenamento, os medicamentos foram registrados em $76,7 \%$ dos casos, e a intenção suicida foi relatada entre 25,6\% (AL-BARRAQ; FARAHAT, 2011). Estudo envolvendo 473 casos de envenenamentos acidentais entre menores de 15 anos residentes em Londrina, Paraná, Brasil, em 2001, mostrou que $47,5 \%$ ocorreram por medicamentos, seguidas por pesticidas $(14,1 \%)$ e produtos de limpeza $(11,3 \%)$ (MARTINS et al., 2006).

Os medicamentos ocupam a primeira posição no conjunto dos agentes tóxicos considerados (MORAIS etal., 2008). Em 2008, as intoxicações medicamentosas foram responsáveis pela ocorrência de 30,7\% das intoxicações registradas pelo Sistema Nacional de Informações Tóxico Farmacológicas (SINITOX) da Fundação Oswaldo Cruz. Segundo este levantamento, as intoxicações medicamentosas mais frequentes decorreram de tentativas de suicídio e acidentes individuais (BERTASSOBORGES et al., 2010). Há geralmente um grande número de medicamentos nos domićlios e que podem ser comprados nos balcões das farmácias. Ao explorar a epidemiologia e prevenção de envenenamento acidental de casa em casa, verifica-se que em conformidade com estudos publicados anteriormente, o envenenamento acidental é largamente prevalente na faixa etária de um a cinco anos, com poucos casos em crianças com idade inferior a um ano, quando sua capacidade de explorar é limitada, ou após a idade de cinco anos, presumivelmente porque há capacidade discriminativa com o que ingerem (MENA et al., 2004).

No município de Feira de Santana, Bahia, Brasil, nos anos de 2007 a 2010, as intoxicações notificadas ao Ceatox que ocorreram por medicamentos representaram $33 \%$ das intoxicações, seguida de "raticidas" (18\%), sendo a "tentativa de suicídio", sendo o sexo feminino responsável pelo maior número de casos registrados, assim como as crianças de $1 \mathrm{a} 4$ anos e os adultos jovens, em concordância com os nossos resultados (TELES et al., 2013), porém no referido estudo, observou-se que a tentativa de suicídio foi a circunstância responsável, em 2010 , por $81 \%$ das notificações, dado que excede os resultados encontrados no presente estudo.

Estudo retrospectivo semelhante realizado no Chile com registros de chamadas para informações toxicológicas recebidas durante o período 1992-2002 mostrou que a principal circunstância de exposição foi "não intencional" (78,6\%), com intoxicações em crianças menores de 5 anos motivando $50 \%$ das chamadas. No referido estudo, também os medicamentos foram as substâncias mais comuns, respondendo por $49,2 \%$ das chamadas, seguido por produtos de limpeza $(12,1 \%)$, pesticidas $(11,3 \%)$ e de produtos químicos $(10,5 \%)$ e cosméticos (2,7\%) (MENA et al., 2004).

Dentre estes acidentes, as intoxicações são consideradas de maior crescimento, devido ao desenvolvimento da indústria química e farmacêutica, o uso irracional de medicamentos, ao largo emprego de praguicidas, à prescrição médica abusiva de medicamentos controlados, falha de cuidado adequado no manuseio de substâncias tóxicas, além da facilidade de obtenção das mesmas.

De acordo com os dados observados no estudo atual, uma pesquisa realizada na Turquia mostrou que a taxa de intoxicações foi mais elevada em pessoas de 16-25 anos, a maioria do sexo feminino (68,8\%), e a ingestão oral foi responsável por $78,8 \%$ dos casos, observando-se taquicardia e náuseas e vômitos entre os sintomas mais frequentes. Contudo, ao contrário do que foi observado no presente estudo, a causa comum de intoxicação foi deliberada $(58,6 \%)$, seguida pelo envenenamento não intencional (39,1\%) (GÜLOĞLU et al., 2005).

É importante lembrar que a notificação dos casos de forma correta, envolve diretamente o preenchimento adequado da ficha de notificação, as quais contribuem para o melhor entendimento epidemiológico dos casos ocorridos tanto na capital como em cidades adjacentes do interior, bem como minimizando os casos subnotificados. Além disso, o trabalho mostra a importância dos registros de casos para medidas estratégicas, sejam elas preventivas e profiláticas a serem realizadas pelos órgãos competentes de saúde e educação da população, contribuindo para o melhor entendimento em Saúde Pública no Estado.

As limitações do presente estudo decorrem da frágil institucionalização dos Ceatox, da falta de padronização dos registros e da heterogeneidade dos bancos de dados utilizados. A alta frequência de casos de evolução não confirmada deve-se à natureza do serviço onde foi feita a pesquisa, haja vista que é de consulta e 
assistência toxicológica aos profissionais de saúde e à população geral, não sendo realizada presencialmente, dificultando a obtenção dos dados e análise do quadro clínico e sua evolução.

Apesar de estes números chamarem atenção, sabe-se que há subnotificação, em virtude de casos de intoxicações assintomáticas e as com quadro clínico leve, que nem sempre chegam ao serviço de saúde, e que não são notificados nos centros de informação toxicológica, considerando que, na maior parte das vezes estes centros são acionados apenas com o objetivo de obter informações de como proceder (MONTEIRO; CARVALHO JUNIOR, 2007). Entre os casos notificados existem diferentes problemas nos Sistemas de Informação em Saúde (SIS) como não identificação de casos crônicos, dados incompletos, inadequados e informações que não possuem capacidade de subsidiar ações.

\section{CONCLUSÃO}

Conclui-se que o padrão epidemiológico das intoxicações exógenas notificadas ao Ceatox-PB em 2012 foi similar ao relatado em outros estudos, em que jovens e mulheres foram os grupos de maior risco e os medicamentos ingeridos de forma acidental, os principais causadores.

\section{REFERÊNCIAS}

AL-BARRAQ, A.; FARAHAT, F.S. Pharmaceutical Journal Pattern and determinants of poisoning in a teaching hospital in Riyadh. Saudi J Biological Sciences, v. 19, p. 57-63. 2011.

ANTHONY, L.; KULKARNI, C. Patterns of poisoning and drug overdosage and their outcome among in-patients admitted to the emergency medicine department of a tertiary care hospital. Indian J Crit Care Med. v. 16, p. 130-5, 2012.

BAHLS, S.C; BOTEGA, N.J. Epidemiologia das tentativas de suicídio e dos suicídios. In: MELLO, M.F.; MELLO, A.A.F.; KOHN, R. Epidemiologia da saúde mental no
Brasil. Porto Alegre: Artmed, 2007. p. 151-71.

BARRETO, M.D.L.; MARTINS, M.L.; DINIZ, M.K.L.; FELIX, R.A.; PEREIRA, L.R.; FERNANDES, M.C.A. Perfil das Intoxicações por Tentativas de Suicídio nos 15 Municípios Assistidos Pela $9^{\circ}$ Gerência de Saúde do Estado da Paraíba. Rev Interdisc Saúde, v.2, n.4, p. 393-395, 2015.

BERTASSO-BORGES, M.S.; RIGETTO,J.G.; FURINI, A.A.C.; GONÇALVES, R.G. Eventos toxicológicos relacionados a medicamentos registrados no CEATOX de São José do Rio Preto, no ano de 2008. Arq Ciênc Saúde, v. 17, n. 1, p. 35-41, 2010.

FUNDAÇÃO OSWALDO CRUZ. Sistema Nacional de Informações Tóxico-Farmacológicas. Casos registrados de intoxicação humana. Rio de Janeiro: Fiocruz, 2010. Disponível em http://www.fiocruz.br/sinitox, acessado em $02 /$ nov/2013.

GANDOLFI, E.; ANDRADE, M.G.G. Eventos toxicológicos relacionados a medicamentos no Estado de São Paulo. Rev Saúde Pública, v. 40, n. 6, p. 1056-64, 2006.

GÜLOĞLU, C.; KARA, I.H. Acute poisoning cases admitted to a university hospital emergency department in Diyarbakir, Turkey. Hum Exp Toxicol., v. 24, p. 49-54, 2005.

JESUS, H.S.; BELTRÃO, H.B.M.; ASSIS, D.M. Avaliação do sistema de vigilância das intoxicações exógenas no âmbito da saúde do trabalhador no Brasil entre 2007 e 2009. Cad. Saúde Colet., v. 20, n. 4, p. 515-24, 2012.

JIMÉNEZ, M.R.; KUHN, G.R. Toxicología Fundamental. 4. ed. Servilla: Díaz de Santos, 2009.

KUMAR, S.V.; VENKATESWARLU, B.; SASIKALA, M.; KUMAR, G.V. A study on poisoning cases in a tertiary care hospital. J Nat Sci Biol Med., v. 1, n. 1, p. 35-9. 2010.

MARTINS, C.B.G.; ANDRADE, S.M.; PAIVA, P.A.B. Envenenamentos acidentais entre menores de 15 anos em município da Região Sul do Brasil. Cad. Saúde Pública., v. 22, n. 2, p. 407-414, 2006. 
MENA, H.; CRISTIÁN BETTINI, S. et al. Epidemiología de las intoxicaciones en Chile: una década de registros. Rev. méd. Chile. v.132, n.4, p. 493-499, 2004.

Recebido em:02 março de 2016

MENDONÇA, R.T.; MARINHO, J.L. Discussão sobre Intoxicações por Medicamentos e Agrotóxicos no Brasil de 1999 a 2002. Rev Eletr Farmácia. v. 2, n. 2, p. 45-63, 2005.

MONTEIRO, P.A.A.; CARVALHO JUNIOR, P.M. Características epidemiológicas dos atendimentos de intoxicações humanas no CEATOX-79 (Marília-S.P.) em 2004. Rev. Bras. Toxicol., v. 20, n. 1,2, p. 39-45, 2007.

MORAIS, I.C.O.; BRITO, M.T.; MARIZ, S.L.; FOOK, S.M.L.; RABELLO, I.P.; OLIVEIRA, F.N. Perfil epidemiológico das intoxicações medicamentosas registradas pelo Centro de Assistência e Informação Toxicológica de Campina Grande (PB) no período de 2005 a 2007. Rev. Bras. Farm., v. 89, n. 4, p. 356-352, 2008.

MOREIRA, C.S.; BARBOSA, N.R.; VIEIRA, R.C.; CARVALHO, M.R. de; MARANGON, P.B.; SANTOS, P.L. et al. A retrospective study of intoxications admitted to the university hospital/UFJF from 2000 to 2004. Cienc Saude Colet., v. 15, p. 879-88, 2010.

SILVA, P.M.G.; SILVA, D.G.; PONCE DE LEON, C.E.J.; LEITE, F.F.; BRUNS, S.F.; MAGALHAES, H.I.F. O Centro de Assistência Toxicológica - Ceatox na Escola Pública: Ferramenta de Aprimoramento Acadêmico e Fonte de Informação para a Comunidade. In: ENCONTRO DE EXTENSÃO DA UNIVERSIDADE FEDERAL DA PARAÍBA, 16, 2015, João Pessoa. Anais... João Pessoa: UFPB, 2015.

TELES, A.S.; OLIVEIRA, R.F.A.A.; COELHO, T.C.B.; RIBEIRO, G.V.; MENDES, W.M.L.; SANTOS, P.N.P. Papel dos medicamentos nas intoxicações causadas por agentes químicos em município da Bahia, no período de 2007 a 2010. Rev Ciênc Farm Básica Apl., v. 34, n. 2, p. 281288, 2013.

VIANA, G.N.; ZENKNER, F.M.; SAKAE, T.M.; ESCOBAR, B.T. Prevalência de suicídio no sul do Brasil, 2001-2005. J

Bras Psiquiatr., v. 57, n. 1, p. 38-43, 2008. 